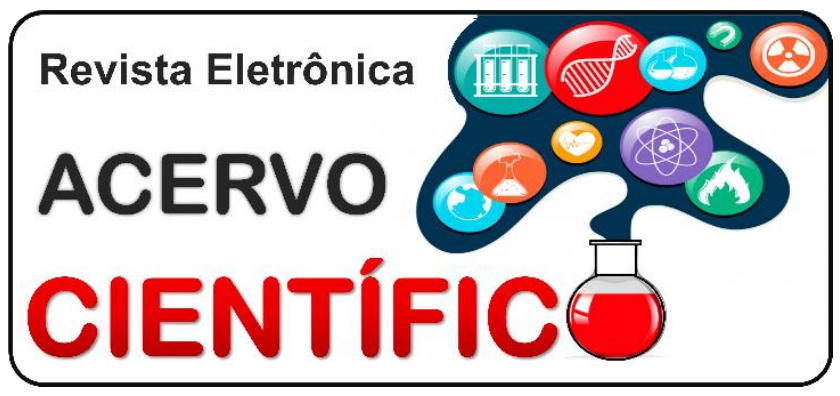

ARITGO ORIGINAL

Recebido em: 3/2020

Aceito em: $3 / 2020$

Publicado em: 6/2020

\title{
Análise retrospectiva sobre quantitativo de cirurgias de vasectomia versus laqueadura tubária no estado de Sergipe entre 2008 e 2019
}

\author{
Retrospective analysis on the quantitative of vasectomy surgeries versus tubular tubing in \\ the state of Sergipe between 2008 and 2019
}
Análisis retrospectivo sobre la cuantitativa de cirugías de vasectomía versus tubular en el estado de Sergipe entre 2008 y 2019

Roberta Souza Santos ${ }^{1 *}$, Manuela Florence Carvalheira Gomes ${ }^{2}$, Ariane Andreza Souza Santos de Oliveira ${ }^{1}$, Amanda Silveira de Carvalho Dantas ${ }^{1}$, Mayana Lula Andrade ${ }^{1}$, Rebeca dos Santos Sirqueira ${ }^{1}$, Roberto Santos Júnior ${ }^{3}$, Alanna Rebeca Teles dos Santos ${ }^{1}$, Marcos Vinícius Costa Menezes ${ }^{1,4}$.

\begin{abstract}
Resumo: Esse artigo buscou realizar uma análise retrospectiva do quantitativo de laqueadura e vasectomias realizadas no estado de Sergipe de 2008 a 2019. Trata-se de uma análise quantitativa, descritiva e retrospectiva com dados colhidos no Sistema de Informação Hospitalar do Departamento de Informática do Sistema Único de Saúde referente a todos os pacientes submetidos à vasectomia e laqueadura tubária em Sergipe de 2008 a 2019. Realizou-se 20.358 laqueaduras e 3.706 vasectomias neste intervalo de tempo estudado. Quanto aos gastos com serviços hospitalares, mostrou-se uma variação de $184.303,33$ a 532.135,96 reais, enquanto a vasectomia foi entre $21.106,60$ e $57.712,97$ reais. Em relação à permanência hospitalar, a cirurgia de laqueadura oscilou de 1,3 dias ( $31 \mathrm{~h}$ e 12 minutos) a 1,7 dias ( $40 \mathrm{~h}$ e 48 minutos) ao passo que a de vasectomia obteve tempo entre 0,2 dias (4h e 48 minutos) e 0,7 dias (16h e 48 minutos). Ocorreram 2 óbitos por laqueadura e nenhum por vasectomia. Durante este período explorado, conclui-se um maior quantitativo de cirurgias de laqueadura em relação às de vasectomia, assim como maior tempo de internação. Ademais, no quesito complicações, observou-se que um maior percentual de óbitos nas laqueaduras em detrimento das vasectomias.
\end{abstract}

Palavras-chave: Laqueadura, Vasectomia, Métodos contraceptivos.

Abstract: This article sought to carry out a retrospective analysis of the amount of ligation and vasectomies performed in the state of Sergipe from 2008 to 2019. This is a quantitative, descriptive and retrospective analysis, developed from the collection in the database provided by the Hospital Information System of the Informatics Department of the Unified Health System regarding vasectomy and tubal ligation procedures from 2008 to 2019. The present study included all patients who underwent vasectomy and tubal ligation surgery in Sergipe. 20,358 tubal ligations and 3,706 vasectomies were performed in the studied period. As for spending on hospital services, there was a variation of $184,303.33$ to $532,135.96$ reais, while the vasectomy was between $21,106.60$ and $57.712,97$ reais. Regarding hospital stay, the ligation surgery ranged from 1.3 days ( $31 \mathrm{~h}$ and 12 minutes) to 1.7 days ( $40 \mathrm{~h}$ and 48 minutes), while the vasectomy surgery took

\footnotetext{
1 Universidade Tiradentes (UNIT), Aracaju - SE. *E-mail: robertasouzamed@gmail.com

2 Centro Universitário Jorge Amado (UNIJORGE), Salvador - BA.

${ }^{3}$ Universidade Federal de Sergipe, Aracaju - SE.

${ }^{4}$ Universidade Federal de Sergipe (UFS), Lagarto - SE.
} 
between 0.2 days ( $4 \mathrm{~h}$ and $48 \mathrm{~h}$ ) minutes) and 0.7 days ( $16 \mathrm{~h}$ and 48 minutes). There were 02 deaths due to tubal ligation and none due to vasectomy. During this explored period, a greater number of tubal ligation surgeries is concluded in relation to those of vasectomy, as well as a longer hospital stay. Furthermore, in terms of complications, it was observed that a higher percentage of deaths in the tubal ligation to the detriment of vasectomies.

Keywords: Tubal ligation, Vasectomy, Contraceptive methods.

Resumen: Este artículo buscó realizar un análisis retrospectivo de la cantidad de ligaduras y vasectomías realizadas en el estado de Sergipe de 2008 a 2019. Este es un análisis cuantitativo, descriptivo y retrospectivo, desarrollado a partir de la recopilación en la base de datos proporcionada por el Sistema de información hospitalaria del Departamento de Informática del Sistema Único de Salud con respecto a los procedimientos de vasectomía y ligadura de trompas de 2008 a 2019. El presente estudio incluyó a todos los pacientes que se sometieron a cirugía de ligadura de trompas y vasectomía en Sergipe. Se realizaron 20.358 ligaduras de trompas y 3.706 vasectomías en el período estudiado. En cuanto al gasto en servicios hospitalarios, hubo una variación de 184.303,33 a 532.135,96 reales, mientras que la vasectomía fue de entre $21.106,60$ y $57.712,97$ reales. Con respecto a la estadía en el hospital, la cirugía de ligadura varió de 1.3 días (31h y 12 minutos) a 1.7 días (40h y 48 minutos), mientras que la cirugía de vasectomía tomó entre 0.2 días (4h y $48 \mathrm{~h}$ ) minutos) y 0,7 días (16h y 48 minutos). Hubo 02 muertes por ligadura de trompas y ninguna por vasectomía. Durante este período explorado, se concluye un mayor número de cirugías de ligadura de trompas en relación con las de la vasectomía, así como una estadía hospitalaria más prolongada. Además, en términos de complicaciones, se observó que un mayor porcentaje de muertes en la ligadura de trompas en detrimento de las vasectomias.

Palabras clave: Ligadura de trompas, Vasectomía, Métodos anticonceptivos.

\section{INTRODUÇÃO}

Ao longo dos anos, percebe-se uma tendência em centralizar o controle da natalidade sob uma perspectiva da figura feminina. Esse pensamento sempre se fez presente, convivendo, no contexto dos anos 70 , com os conceitos do planejamento familiar que reforçava os benefícios do controle da prole sob a responsabilidade feminina, sendo apontadado como um bem a saúde da mulher, da família e da sociedade em geral. Em meados dos anos 80 , a definição de saúde reprodutiva se tornou mais amplo, dando maior abrangência às relações de gênero que estão engessadas no exercício dos direitos reprodutivos, passando a inserir a ideologia de que o controle da prole deve ser abordado tanto em relação às mulheres como também deverá ser abordado com os homens concomitantemente, colocando a responsabilidade do controle da prole familiar em igual responsabilidade para homens e mulheres (BARZELATTO J e HEMPEL M, 1990; ALVARENGA AT e SCHOR N, 1998; OSIS MJD, 1998; ALCALÁ MJ, 1995).

À proporção em que a mulher tem efetivamente participado mais do mundo público, o homem vem sendo requisitado a ser mais participativo nas vivências familiares com maior participação nos trabalhos domésticos e no cuidado com os filhos. Sendo assim, um novo conceito de papel familiar sobre o homem está emergindo, porém num processo gradual e paulatino (CARVALHO CC, 2016).

Em 1997, o Ministério da Saúde, por meio da Portaria №144 e, posteriormente, da Portaria ํo 048 incluiu a laqueadura tubária e a vasectomia no grupo de procedimentos cirúrgicos do Sistema Único de Saúde (SUS), permitindo a esterilização nas seguintes situações: no caso de homens e mulheres maiores de 25 anos ou com pelo menos dois filhos vivos, e no caso de risco para a mãe ou para o filho, tendo testemunhado em relatório escrito e assinado por dois médicos, observando um prazo mínimo de 60 dias entre a manifestação da vontade do casal e a realização da cirurgia (BRASIL, 1996).

A saúde reprodutiva está inserida no Programa de Planejamento Familiar e os serviços de saúde devem disponibilizar aos usuários, homens e mulheres, ações educativas individuais ou em grupo, garantir o acesso a informações, meios contraceptivos existentes, métodos e técnicas disponíveis para controle da fecundidade que não prejudiquem a vida e a saúde das pessoas, assegurando direitos iguais para homens, mulheres ou casal, sendo a escolha do método livre e informada (BRASIL, 2010). 
Sendo assim, esse trabalho visa analisar, retrospectivamente, através de dados estatísticos o quantitativo de vasectomia em relação ao quantitativo de laqueadura tubária realizado pela rede pública de saúde no estado de Sergipe.

\section{MÉTODOS}

Trata-se de um estudo quantitativo, descritivo e retrospectivo, elaborado a partir de uma detalhada coleta e análise de dados através do Sistema de Informações Hospitalares do departamento de informática do Sistema Único de Saúde do Brasil (SIH - SUS - DATASUS). Os códigos utilizados nesta pesquisa foram 0409040240 e 0409060186, relacionados a procedimentos de vasectomia e laqueadura tubária, respectivamente. Este artigo inclui todos os pacientes submetidos à laqueadura e vasectomia do estado de Sergipe e atendidos pela atenção pública de saúde do país no período de 2008 a 2019.

Os dados coletados foram tabulados em uma planilha confeccionada no programa Microsoft Excel e categorizados por ano, por procedimento realizado. Foi realizada, então, uma análise descritiva caracterizando as variáveis categóricas por meio de frequência absoluta e relativa. Vale ressaltar que, a presente pesquisa não necessitou ser submetida à aprovação do Comitê de Ética em Pesquisa (CEP) por se tratar de informações contidas em um banco de dados de domínio público e acessível a qualquer cidadão.

\section{RESULTADOS E DISCUSSÃO}

Esse atual estudo constatou que, durante o período analisado, o procedimento de laqueadura tubária obteve maior número $(n=20.358)$ em relação à vasectomia $(n=3.706)$, como mostra na tabela 1 , onde expõe o número total de internações pelo SUS dos pacientes que foram submetidos a ambos os procedimentos analisados.

O total de procedimentos realizados de laqueaduras correspondeu a $84,60 \%$ enquanto os de vasectomia a $15,40 \%$. Vale ressaltar que não estão inclusos os casos de laqueadura das pacientes que a realizaram concomitantemente ao parto cesáreo.

Tabela 1 - Número total de internações para realização de laqueadura e vasectomia distribuída por ano em Sergipe.

\begin{tabular}{ccc}
\hline Ano & Laqueadura & Vasectomia \\
\hline 2008 & 2915 & 393 \\
2009 & 1585 & 192 \\
2010 & 1708 & 138 \\
2011 & 2222 & 309 \\
2012 & 2651 & 447 \\
2013 & 1461 & 333 \\
2014 & 1915 & 339 \\
2015 & 1515 & 305 \\
2016 & 1457 & 269 \\
2017 & 920 & 303 \\
2018 & 1006 & 381 \\
2019 & 1003 & 297 \\
\hline Total & 20.358 & 3.706 \\
\hline
\end{tabular}

Fonte: Santos RS, et al., 2020; dados extraídos de Ministério da Saúde Sistema de Informações Hospitalares do SUS (SIH/SUS), 2019.

Percebe-se, diante desses dados, um declínio no número de cirurgias de laqueadura ao longo dos anos, totalizando 2915 casos em 2008 com redução para 1003 casos em 2019. Observou-se que este declínio não é acompanhado do aumento das vasectomias realizadas já que estas se mantêm em valores não muito discrepantes entre os anos, exceto no ano de 2009 e 2010, onde observamos valores mais baixos, 192 e 138 , respectivamente. 
A vasectomia é um dos poucos métodos contraceptivos disponibilizado ao público masculino, e com grande adesão em países desenvolvidos, como Austrália, Canadá, Holanda e Estados Unidos, porém apresenta baixa adesão na maioria dos países em desenvolvimento, incluindo os da América Latina (POTTS JM, et al., 1999; PILE JM e BARONE MA, 2009).

Em contrapartida, a prevalência de esterilização feminina cresceu e, atualmente, é o método contraceptivo de maior adesão entre as mulheres em idade fértil. Em geral, os homens detém predomínio nas decisões da vida familiar, porém no aspecto de anticoncepção, a responsabilidade é colocada sob a mulher e, nesse contexto, o homem só se sensibiliza a interferi na decisão para controle da prole quando há necessidade de um método mais definitivo como a esterilização (MARCHI EM, et al., 2003).

No Brasil, ainda é recente a participação masculina ativa na escolha pelos métodos contraceptivos, sobretudo em regiões mais afastadas das capitais metropolitanas, onde o acesso à educação e aos serviços de saúde é mais difícil, levando a falta de informação e de atendimentos para essa população (KAVANAUGH ML E ANDERSON RM, 2013).

O público alvo da atenção em planejamento familiar são homens, mulheres e casais, porém, o programa de planejamento familiar tende a priorizar as mulheres, uma vez que, historicamente, a mulher tem sido colocada na responsabilidade de controle da prole e os métodos contraceptivos disponibilizados pelo Ministério da Saúde são, em sua maioria, destinados para as mulheres (CARVALHO CC, 2016).

A esterilização masculina tem se tornado cada vez mais alvo da escolha de muitos casais, como método contraceptivo, apesar de todos os estereótipos em relação às causas e efeitos da vasectomia, pois muitos homens tem demonstrado desconhecimento em relação ao procedimento cirúrgico, sendo relatada entre alguns homens a preocupação com possível alteração da virilidade e libido após o procedimento da vasectomia (CARVALHO CC, 2016).

Para Ferreira RV, et al. (2014) dentre os fatores que explicam a atuação mais efetiva da população masculina no planejamento familiar está envolvido aspectos histórico e cultural, uma vez que os antepassados inferem que ter filhos é um sinal de virilidade masculina. Além disso, Ferreira RV, et al. (2014), infere que os homens são vistos como protagonistas na economia e política, sendo destinadas as mulheres o papel nas atividades domésticas e reprodutivas, ou seja, não são aptas à esfera pública, entendendo que à natureza feminina cabe o cumprimento da maternidade na sociedade.

No geral, as pesquisas vêm apontando que o tempo de espera entre a solicitação e a cirurgia há indícios de diferenças de gênero no atendimento, sendo menor a espera dos homens que serão submetidos a vasectomia do que em relação as mulheres que farão laqueadura. Mais frequentemente que as mulheres, os homens conseguem fazer a cirurgia antes que se passassem os necessários sessenta dias de carência (OSIS MJD, et al., 2003; OSSIS MJD, et al., 2006).

Não existe evidência de que a vasectomia curse com impotência sexual no paciente, embora existam relatos de casos de disfunção erétil grave associada a problemas psicológicos após procedimento da vasectomia e não estão relacionados ao procedimento cirúrgico. Antes da realização da vasectomia é realizado atendimento ao casal com psicólogo, assistente social ou enfermeiro para esclarecimentos visando atenuar medos e temores (PROJETO DIRETRIZES, 2009)

É coerente que tenhamos em mente que o foco da questão não se baseia em inverter a estatística de esterilização feminina, aumentando a esterilização masculina, mas vai muito além disso, através de mudança na perspectiva acerca dos meios contraceptivos, ampliando as informações inerentes a cada método, afim de que tanto homens como mulheres possam ter o livre arbítrio na escolha do método que julgar mais adequado pra si, levando - se em consideração suas motivações, perspectivas e expectativas quanto à contracepção (MARCHI EM, et al., 2003).

A respeito dos gastos totais de serviços hospitalares de laqueadura e vasectomia, a Tabela 2 apresenta estes dados e o valor unitário de cada tipo de procedimento categorizado por ano. 
Tabela 2 - Valor serviço hospitalares do procedimento e valor por procedimento por ano em Sergipe.

\begin{tabular}{|c|c|c|c|c|}
\hline Ano & Laqueadura & Valor AlH por laqueadura & Vasectomia & Valor AlH por vasectomia \\
\hline 2008 & $492.303,01$ & 295,93 & $57.712,97$ & 226,28 \\
\hline 2009 & $318.274,29$ & 339,81 & $33.364,80$ & 276,81 \\
\hline 2010 & $342.918,54$ & 339,76 & $21.106,60$ & 317,48 \\
\hline 2011 & $445.842,33$ & 339,65 & $41.195,88$ & 313,26 \\
\hline 2012 & 532.135 .96 & 339,75 & $59.658,04$ & 316,03 \\
\hline 2013 & $292.955,71$ & 358,65 & $44.419,56$ & 322,07 \\
\hline 2014 & $387.421,52$ & 346,76 & $45.195,48$ & 324,69 \\
\hline 2015 & $307.004,68$ & 342,21 & $40.790,60$ & 323,82 \\
\hline 2016 & $294.607,75$ & 341,18 & $36.007,08$ & 320,84 \\
\hline 2017 & $184.303,33$ & 339,33 & $40.467,96$ & 313,70 \\
\hline 2018 & $201.338,87$ & 339,12 & $51.154,92$ & 317,79 \\
\hline 2019 & $201.089,05$ & 339,48 & $40.220,04$ & 334,76 \\
\hline
\end{tabular}

Fonte: Santos RS, et al., 2020; dados extraídos de Ministério da Saúde - Sistema de Informações Hospitalares do SUS (SIH/SUS), 2019. 
Percebe-se que há um gasto maior com serviços hospitalares da laqueadura em relação à vasectomia. $\mathrm{A}$ laqueadura atinge um valor total mínimo com serviços hospitalares de $184.303,33$ reais e valor total máximo de 532.135,96 reais, enquanto a vasectomia obteve valor total mínimo de 21.106,60 reais e, máximo de $57.712,97$ reais. Nota-se ainda que o valor unitário da AlH para laqueadura e valor unitário da AlH para vasectomia apresentam valores próximos tendo diferença de valor unitário da laqueadura em relação ao valor unitário da vasectomia, diferença máxima de 69,65 em 2008 e diferença mínima de 4,72 em 2019.

Apenas os serviços do SUS devidamente certificados podem receber reembolso para os procedimentos hospitalares de laqueadura tubária e vasectomia. Durante o desenvolvimento do programa de planejamento familiar, alguns obstáculos foram surgindo para sua efetivação, como: demora entre solicitação e a realização do procedimento, proibição da laqueadura no parto e pós-parto, além de cobertura territorial limitada de serviços médicos do SUS credenciados (CAETANO AJ, 2014).

A vasectomia é mais simples, mais segura, mais fácil de ser realizada e mais barata que a esterilização feminina. Além disso, a vasectomia é executada sob anestesia local. Ressalta - se que apesar da vasectomia ser mais simples, ambos os métodos de esterilização (vasectomia e laqueadura), são seguros e permanentes para casais que decidiram não ter filhos, sendo a decisão unicamente restrita ao casal (OMS, 2007).

Alguns homens relatam que após a vasectomia tem aumento no prazer e frequência nas relações sexuais. O método contraceptivo da vasectomia é seguro, permanente e prático. A reversibilidade tanto da vasectomia como da laqueadura é muito difícil e onerosa, difícil e está indisponível na maioria das regiões. Um fator adicional, é que a vasectomia tem um papel importante ao tornar o homem também responsável pela contracepção, minimizando a responsabilidade atribuída à mulher (OMS, 2007).

Embora a OMS infira que a vasectomia seja mais barata que a laqueadura, é possível avaliar através da tabela 2 que, de fato, o valor unitário entre laqueadura e vasectomia não são tão discrepantes, mas quando se analisa o custo total com gastos hospitalares, percebemos que o valor da laqueadura incluindo valor de procedimento e gastos hospitalares, torna-se mais dispendioso, visto que, um dos aspectos que contribui para isso é o tempo de permanência hospitalar dispensado à laqueadura em detrimento a vasectomia como observado na Tabela 3.

Quando se analisa o tempo de permanência hospitalar, percebe-se que há maior permanência hospitalar relacionada à cirurgia de laqueadura tubária em detrimento a cirurgia de vasectomia. A cirurgia de laqueadura variou entre 1,3 dias ( $31 \mathrm{~h}$ e 12 minutos) na maioria dos anos com o intervalo de tempo máximo de 1,7 dias (40h e 48 minutos) em 2018. Já a vasectomia, obteve tempo de permanência hospitalar mínimo de 0,2 dias (4h e 48 minutos) em 2008 e máximo de 0,7dias (16h e 48 minutos) nos anos de 2010 e 2019, conforme consta na Tabela 3.

Tabela 3 - Tempo médio de permanência hospitalar distribuído por ano.

\begin{tabular}{ccc}
\hline Ano & Dias/laqueadura & Dias/Vasectomia \\
\hline 2008 & 1,3 & 0,2 \\
2009 & 1,4 & 0,5 \\
2010 & 1,4 & 0,7 \\
2011 & 1,3 & 0,6 \\
2012 & 1,3 & 0,5 \\
2013 & 1,3 & 0,4 \\
2014 & 1,3 & 0,5 \\
2015 & 1,3 & 0,5 \\
2016 & 1,4 & 0,5 \\
2017 & 1,4 & 0,3 \\
2018 & 1,7 & 0,3 \\
2019 & 1,4 & 0,7 \\
\hline
\end{tabular}

Fonte: Santos RS, et al., 2020; dados extraídos de Ministério da Saúde - Sistema de Informações Hospitalares do SUS (SIH/SUS), 2019. 
A vasectomia envolve um procedimento cirúrgico simples e seguro. De modo geral, o paciente pode sair da clínica em uma hora (OMS, 2007). A recomendação pós-vasectomia é de repouso e abstinência sexual por 2 a 3 dias, a cirurgia ocorre sob anestesia local e é realizada uma pequena incisão em região do escroto e através dessa incisão irá realizar o fechamento do canal deferente (OMS, 2007).

A laqueadura é realizada sob anestesia local com sedação moderada e em algumas situações poderá evoluir para anestesia geral. Após a cirurgia a paciente terá que evitar trabalho vigoroso ou levantar peso por 1 semana, abstinência sexual por pelo menos 1 semana, em persistindo dor, a abstinência sexual poderá ser prolongada até cessação da dor (OMS, 2007).

A cirurgia de laqueadura, geralmente é realizada com obstrução ou secção das tubas uterinas para impedir a passagem do óvulo e, consequentemente, sua fertilização (HOFFMAN BL, et al., 2014). Durante o procedimento a mulher poderá ficar acordada, sendo possível a equipe oferecer apoio emocional, tranquilizando-a ou poderá ser utilizado anestésico e sedativos, ajustados ao peso corporal da paciente, porém, deve-se evitar sedação excessiva, pois poderá reduzir a consciência da paciente ou gerar complicações na dinâmica respiratória (OMS, 2007).

Há três métodos utilizados para ligadura tubária. Tais métodos incluem aplicação de diversos anéis ou clipes permanentes às tubas uterinas, eletrocoagulação de um segmento ou ligadura propriamente dita com fio de sutura, com ou sem remoção adicional de um segmento da tuba uterina (HOFFMAN BL, et al., 2014).

A cirurgia de laqueadura poderá ainda ser realizada através de uma mini laparatomia onde se fará uma incisão horizontal de 2-5 centímetros em região suprapúbica ou poderá ser via laparoscópica em que uma agulha especial é colocada no abdômen e através dela é inflado gás para elevar a parede abdominal e afastar os órgãos (OMS, 2007).

A esterilização feminina é um método de contracepção seguro. Contudo, requer cirurgia e anestesia, as quais apresentam alguns riscos tais como infecção ou abscesso da ferida. Complicações graves são incomuns. Óbito, devido ao procedimento ou à anestesia, é extremamente rara. O risco de complicações com anestesia local é significativamente menor do que com anestesia geral. As complicações podem ser mantidas num patamar mínimo se forem utilizadas técnicas apropriadas e se o procedimento for executado em local adequado (PROJETO DIRETRIZES, 2009).

Em se tratando de métodos contraceptivos de caráter definitivo e por ser procedimento em que há pouco acesso às técnicas de reversibilidade da cirurgia, o casal deverá ser orientado sobre outros métodos contraceptivos que não são permanentes, bem como deverá ser esclarecido sobre complicações que poderão ocorrer e não somente informar a eficácia da cirurgia. Além das informações inerentes ao procedimento, o acolhimento engloba aspectos éticos e legais sendo necessária a assinatura do casal do termo de consentimento esclarecido (BRASIL, 2010).

A laqueadura tubária é o método contraceptivo de maior adesão entre as mulheres, seguido pelo anticoncepcional oral. Mais da metade das cirurgias de laqueadura, no Brasil, é realizada concomitantemente ao parto cesáreo, o que eleva o quantitativo de partos cirúrgicos. Quando analisamos os casos de vasectomias, percebe-se que são em número absolutos o método contraceptivo com menor adesão (BRASIL, 2010).

Analisando os dados, percebemos que a cirurgia de vasectomia apresenta uma série de vantagens em relação à laqueadura tubária, sendo a primeira mais simples e processo de recuperação mais célere que a segunda.

Conforme a Tabela 4 percebe-se que não houve óbitos relacionados à cirurgia de vasectomia no período estudado. Enquanto nas cirurgias de laqueadura tubária, ocorreu 01 óbito no ano de 2010 e outro no ano de 2015, totalizando 02 óbitos por laqueadura no período estudado. 
Tabela 4 - Total de óbitos por laqueadura e por vasectomia realizada distribuída por ano em Sergipe.

\begin{tabular}{ccc}
\hline Ano & Óbitos por laqueadura & Óbitos por vasectomia \\
\hline 2008 & 0 & 0 \\
2009 & 0 & 0 \\
2010 & 1 & 0 \\
2011 & 0 & 0 \\
2012 & 0 & 0 \\
2013 & 0 & 0 \\
2014 & 0 & 0 \\
2015 & 1 & 0 \\
2016 & 0 & 0 \\
2017 & 0 & 0 \\
2018 & 0 & 0 \\
2019 & 0 & 0 \\
\hline Total & 2 & 0 \\
\hline
\end{tabular}

Fonte: Santos RS, et al., 2020; dados extraídos de Ministério da Saúde - Sistema de Informações Hospitalares do SUS (SIH/SUS), 2019.

A vasectomia é considerada um dos métodos anticoncepcionais mais efetivos e seguros com baixa mortalidade, sendo de 0,1 por 100.000 procedimentos em países industrializados. Após a cirurgia, é necessário que o paciente faça espermograma para confirmar a efetividade da cirurgia que é comprovada pela azoospermia no espermograma (PROJETO DIRETRIZES, 2009).

As complicações podem ser de cunho precoce e tardio. Dentre as complicações precoces está o hematoma que está relacionado à experiência do cirurgião e ao fechamento ou não da incisão cirúrgica, infecção no local da incisão, trato urinário e epididimite, lesão em estruturas adjacentes durante o procedimento, fístulas deferento - cutâneas, bem como poderá desenvolver complicações tardias como granuloma espermático, dor crônica e urolitíase (PROJETO DIRETRIZES, 2009).

A morbidade da cirurgia de laqueadura é indicada pelas taxas de readmissão hospitalar após o procedimento, aumento nos dias de internação e realização de laparotomia secundária à laparoscopia prévia. As complicações associadas ao procedimento são sepse, hemorragia, infarto agudo do miocárdio, embolia pulmonar e complicações anestésicas (hipoventilação principalmente), sendo esta última a principal causa de morte. Paciente com comorbidade associada no momento do procedimento tem maior chance de complicações (PROJETO DIRETRIZES, 2009).

$\mathrm{Na}$ laqueadura alguns fatores relacionados à morbimortalidade estão associados a complicações resultantes de sepse, tromboembolia, dor pélvica, perfuração visceral e alergia ao anestésico (PROJETO DIRETRIZES, 2009). Em se tratando da vasectomia, apresentam-se como procedimento cirúrgico que tem baixíssima mortalidade, as complicações são incomuns ou raras, dentre as raras, a mais comum é a dor aguda no escroto e testículo e muito raramente poderá ocorrer infecção no local da incisão, sendo a vasectomia um procedimento que não há riscos à saúde conhecidos (OMS, 2007).

As gravidezes que ocorrem após esterilização tubária têm alta incidência de implante ectópico em comparação com a taxa encontrada na população geral. Uma outra complicação que poderá ocorrer é a reanastomose espontânea dos segmentos tubários, e portanto, insucesso na laqueadura tubária. A morbidade em geral está associada às readmissões hospitalares após procedimento, aumento nos dias de internação e histórico de laparoscopia anterior ao procedimento (PROJETO DIRETRIZES, 2009).

Pelo exposto, podemos perceber que os riscos inerentes à laqueadura suplantam os da vasectomia, embora os dois procedimentos tenham a mesma finalidade, controle da prole e, consequentemente, planejamento familiar. 


\section{CONCLUSÃO}

Diante deste estudo apresentado, conclui-se que em Sergipe a cirurgia de vasectomia ainda é pouco procurada, principalmente quando comparada à laqueadura. Percebe-se também que os gastos hospitalares com laqueadura são maiores ao comparar com a vasectomia, já que esta última apresenta um tempo de internação hospitalar inferior, não ultrapassando um dia. Faz-se necessário maior atuação e comprometimento da equipe multidisciplinar da atenção básica, no sentido de esclarecer dúvidas e encorajar a população do sexo masculino, a fim de suscitar e inseri-los no Programa Planejamento Familiar, visto que, os homens também têm papel ativo no processo de controle da prole familiar.

\section{REFERÊNCIAS}

1. ALVARENGA AT, SCHOR N. Contracepção feminina e política pública no Brasil: Pontos e contrapontos da proposta oficial. Saúde e Sociedade, 1998; 7:87-110.

2. ALCALÁ MJ. Compromisos para la Salud y los Derechos Sexuales y Reprodutivos de Todos: Marcos de Acción. New York: Family Care International. 1995

3. BARZELATTO J, HEMPEL M. Reproductive Health: A Strategy for the 1990's. New York: Ford Foundation. 1990.

4. BRASIL. MINISTÉRIO DA SAÚDE. Secretaria de Atenção à Saúde. Departamento de Atenção Básica. Saúde sexual e saúde reprodutiva / Ministério da Saúde, Secretaria de Atenção à Saúde, Departamento de Atenção Básica. - Brasília: Ministério da Saúde, 2010.

5. BRASIL. Presidência da República. Casa Civil. Subchefia para Assuntos Jurídicos. Lei no 9.263 de 12 de janeiro de 1996. Brasilia, 15 jan 1996; Seção 1, p.1-3.

6. CAETANO AJ. Esterilização cirúrgica feminina no Brasil, 2000 a 2006: aderência à lei de planejamento familiar e demanda frustrada. Rev. bras. estud. popul., 2014; 31(2):309-331.

7. CARVALHO CC. A Esterilização Masculina: perfil e percepções de homens que optaram pela vasectomia. O social em questão, Ano XIX, n`36, 2016, p.425-446.

8. FERREIRA RV, et al. Planejamento Familiar: Gênero e significados. Textos e contextos, 2014; 13(2): 387 - 397.

9. GALASTRO EP, FONSECA RMGS. A participação do homem na saúde reprodutiva: o que pensam os profissionais de saúde. Revista da Escola de Enfermagem, USP. São Paulo, 2007.

10. HOFFMAN BL, et al. Ginecologia de Williams. 2. ed. Porto Alegre: AMGH, 2014.

11. MARCHI NM, et al. Opção pela Vasectomia e relações de gênero. Caderno de Saúde pública, Rio de Janeiro, 2003.

12. MOREIRA MHC, ARAUJO JNGA. Planejamento Familiar: autonomia ou encargo feminino. Psicologia em Estudo Maringá, 2004; 9(3).

13. MARCHI NM, et al. Opção pela Vasectomia e relações de gênero. Caderno de Saúde pública, Rio de Janeiro, 2003.

14. OSIS MJD, et al. Atendimento à demanda pela esterilização cirúrgica na Região Metropolitana de Campinas, São Paulo, Brasil: percepção de gestores e profissionais dos serviços públicos de saúde. Cad. Saúde Pública, Rio de Janeiro, 2009; 25(3): $625-634$.

15. ORGANIZAÇÃO MUNIDIAL DE SAÚDE (OMS). Planejamento Familiar: um Manual Global para Profissionais e Serviços de Saúde. Genebra: Organização Mundial de Saúde, 2007.

16. OSIS MJD. PAISM: Um marco na abordagem da saúde reprodutiva Brasil. Cadernos de Saúde Pública. 1998; 14(1):25-32.

17. POTTS JM, et al. Patient characteristics associated with vasectomy reversal. The Journal of urology, Baltimore, 1999; 161(6): 1835-1839.

18. PILE JM, BARONE MA. Demographics of vasectomy - USA and international. The Urologic clinic of North America, Philadelphia, 2009; 36(3): 295-305.

19. PROJETO DIRETRIZES. Associação Médica Brasileira (AMB), Conselho Federal de Medicina. (CFM). Esterilização Feminina: Indicação. São Paulo: AMB/CFM; 2009. Disponível em: URL: https://diretrizes.amb.org.br/_BibliotecaAntiga/esterilizacao-feminina-indicacao.pdf.

20. PROJETO DIRETRIZES. Sociedade Brasileira de Medicina de Família e Comunidade.Sociedade, Brasileira de Genética Médica, Sociedade Brasileira de Urologia. Esterilização Masculina: Indicação. São Paulo: AMB/CFM; 2009. Disponível em: https://diretrizes.amb.org.br/_BibliotecaAntiga/esterilizacao-masculinaindicacao.pdf

21. KAVANAUGH ML, ANDERSON RM. Contraception and Beyond: The Health Benefits of Services Provided at Family Planning Centers, New York: Guttmacher Institute, 2013. 